\title{
Retraction
}

\section{Retracted: Knowledge-Based Intelligent Education Recommendation System with IoT Networks}

\author{
Security and Communication Networks
}

Received 16 November 2022; Accepted 16 November 2022; Published 29 November 2022

Copyright $\odot 2022$ Security and Communication Networks. This is an open access article distributed under the Creative Commons Attribution License, which permits unrestricted use, distribution, and reproduction in any medium, provided the original work is properly cited.

Security and Communication Networks has retracted the article titled "Knowledge-Based Intelligent Education Recommendation System with IoT Networks" [1] due to concerns that the peer review process has been compromised.

Following an investigation conducted by the Hindawi Research Integrity team [2], significant concerns were identified with the peer reviewers assigned to this article; the investigation has concluded that the peer review process was compromised. We therefore can no longer trust the peer review process, and the article is being retracted with the agreement of the Editorial Board.

\section{References}

[1] X. Xin, T. Shi, and M. Sohail, "Knowledge-Based Intelligent Education Recommendation System with IoT Networks," Security and Communication Networks, vol. 2022, Article ID 4140774, 10 pages, 2022.

[2] L. Ferguson, "Advancing Research Integrity Collaboratively and with Vigour," 2022, https://www.hindawi.com/post/advancing-re search-integrity-collaboratively-and-vigour/. 


\title{
Knowledge-Based Intelligent Education Recommendation System with IoT Networks
}

\author{
Xin Xin, ${ }^{1}$ Tianlei Shi, ${ }^{2}$ and Mishal Sohail ${ }^{3}{ }^{3}$ \\ ${ }^{1}$ Jinlin Provincial Institute of Education, Jilin, Changchun 130022, China \\ ${ }^{2}$ BOE Technology Group Co., Ltd., Beijing 100010, China \\ ${ }^{3}$ Department of Industrial Engineering, International Ataturk Alatoo University, Bishkek, Kyrgyzstan
}

Correspondence should be addressed to Mishal Sohail; dr.mishalsohail@mail.cu.edu.kg

Received 28 December 2021; Revised 7 January 2022; Accepted 26 January 2022; Published 7 March 2022

Academic Editor: Muhammad Arif

Copyright $\odot 2022$ Xin Xin et al. This is an open access article distributed under the Creative Commons Attribution License, which permits unrestricted use, distribution, and reproduction in any medium, provided the original work is properly cited.

\begin{abstract}
The intelligent education recommendation system can recommend knowledge suitable for students' personal learning. However, the traditional recommendation algorithm has generality problems, which lead to poor knowledge recommendation effects. In order to improve the performance of the education recommendation system, based on the machine learning algorithm, this paper combines the knowledge graph algorithm to improve the recommendation algorithm and decomposes the matrix with a higher dimension into several matrices with relatively small dimensions through matrix transformation. Moreover, this paper conducts in-depth mining of the potential attributes of users and items and improves the matrix decomposition formula based on knowledge recommendation requirements. In addition, this paper constructs the framework of the intelligent education recommendation system with IoT networks based on the analysis of functional requirements. Finally, this paper designs experiments to verify and analyze the model from the perspective of model performance and user satisfaction. The research results show that the algorithm model constructed in this paper is effective.
\end{abstract}

\section{Introduction}

With the rapid development of information technology, more and more system foundations are applied to recommendation technology, and collaborative filtering recommendation algorithms are also used in various fields. Academics have been upgrading classic collaborative filtering recommendation algorithms for a long time to increase recommendation accuracy, create targeted suggestions to users, and obtain customised recommendations. Some academics, for example, combine users' individualised data from diverse sectors of data with collaborative filtering algorithms to increase the accuracy of collaborative filtering suggestions. In addition, researchers are attempting to apply content-based collaborative filtering recommendations to information filtering and other relevant sectors and match and sort user record document information to increase suggestion accuracy [1].
In the era of smart education, personalized education recommendation has become a trend. Content-based recommendation, collaborative filtering recommendation, and mixed recommendation of the two are the basic common algorithms in traditional information recommendation. Traditional information recommendation is mainly based on similar learners or exercises of the same type in the recommended exercises. This kind of recommendation is relatively simple to operate, but the exercise recommendation based on traditional information recommendation does not consider the learner's knowledge point mastery status and the semantic relationship between knowledge, and recommendation cannot be implemented for new users, so the recommendation accuracy is not high [2].

In recent years, the problem of information overload has become more and more serious, and researchers have proposed countless solutions. Among them, recommendation system and search engine are two typical solutions that 
can help users quickly obtain the information they need from Internet information data, which can save users a lot of time and energy. In the mid-1990s, personalized recommendation was first proposed. In the next two decades, the recommendation system developed rapidly and became the focus of attention in academia and industry. Moreover, during this time, a large number of applications and related technologies have been produced. In practical applications, the recommendation system can actively recommend information that may be of interest to the user without the user's active demand. In contrast, search engines require users to actively provide keywords describing their needs to find the information they need, so search engines cannot solve many other needs of users. In a sense, the two are two complementary tools for users. Recommendation systems can be divided into two categories according to different prediction objects. The first category is score prediction, and the other is click rate prediction [3].

\section{Related Work}

As a kind of information filtering technology and tool, the recommender system has been studied by experts and scientific researchers since the 1990s. After years of research and accumulation by scientific researchers, the recommendation system has gradually developed into an independent subject and has achieved many good results in various fields such as academia and industry [4]. According to the literature [5], it is vital to communicate the category of the suggested item to the user in the recommendation system. The item that the user is exploring on the Internet or at the shopping mall, for example, is similar to an item that has been seen before. Collaborative filtering and content suggestion were the mainstays of early recommendation systems. Modeling user or product attribute information, such as product colour, brand, and price, is the goal of content-based recommendation. Content-based recommendations may explicitly explain to the user why this specific item is suggested to them since the item's content information is simpler for them to grasp. The simplest method is to tell the consumer that the suggested item is something he may be interested in. Mao et al. [5] proposed a collaborative filtering algorithm. This method uses the wisdom of the group to overcome the time-consuming problem of the method based on content recommendation when collecting item content information. Zuo et al. [6] proposed an item-based collaborative filtering algorithm which was successfully applied to the shopping mall in Amazon. The recommendation prediction score based on the user model and the recommendation prediction score based on the item model are difficult for users to understand, but they can be understood from a philosophical perspective. For example, for user-based recommendation, we can interpret it as "the items recommended to the user are the items liked by users similar to the user" [7]. Item-based recommendation can be interpreted as "the recommended item is an item similar to the item the user previously liked." The proposed LFM implicit semantic model [8], including the matrix factorization method based on collaborative filtering, has greatly succeeded the recommendation system in scoring prediction. In addition, in recent years, the development of machine learning and deep learning fields has further improved the performance of the recommendation system.

At present, knowledge graph technology has attracted the close attention of researchers and has become an important part of today's intelligent system field. The research field of knowledge graph includes knowledge reasoning expansion system and question answering system, but it is also applied in search engine and related artificial intelligence field [9]. In addition to bringing many ways and ideas to acquire knowledge through the Internet, the knowledge graph is also conducive to knowledge-driven research. Especially, with the advent of the era of big data, mining the hidden theoretical knowledge and methods from big data can transform massive data into meaningful things. In this way, modern analysis technology has gradually transformed into a directed processing mechanism for relevant information [10].

Knowledge graph, big data, and deep learning have all become the core of promoting the development of today's AI technology [11]. In recent years, many international research organizations have joined the research of knowledge graph application platform [12]. They advocated the development of the World Wide Web composed of interconnected documents into a platform for sharing knowledge and resources of interconnected data. Moreover, they launched a platform for large-scale knowledge acquisition, interconnected data analysis, and cross-language knowledge extraction. The platform has been successful in the application fields of government open data resources, smart cities, smart medical care, and so on [13]. In contrast, China started relatively late in this field. At present, most data analysis platforms rely on semantic analysis and data mining. For data processing, using semantic links can better process data information and provide more correct judgments [14]. In the question and answer technology of the knowledge graph, by using the semantic analysis of the question sentence, unstructured questions can be transformed into structured search sentences, and the correct processing method can be found among the existing local databases [15]. This method has very high requirements on the performance of the semantic parser. Moreover, because the granularity of words, phrases, and clauses is different, they are also affected by the ambiguity of text structure and text content. In an environment with a relatively large scale and an open domain knowledge base, the performance usually becomes very low [16]. In recent years, many experts have begun to discuss the question and answer of deep learning knowledge. At present, this method is only limited to simple relationship analysis, and it will be more difficult when faced with complex questions. This is because of the lack of context awareness and the lack of detailed semantic analysis of text content [17]. In terms of semantic search, the knowledge graph corresponds to the search keywords entered by the user to the definition of the objective world object in the knowledge graph. Therefore, the query results directly obtain relevant information that meets the needs of users, rather than a simple web page [18]. 


\section{The Principle of Matrix Factorization}

Definition of eigenvalue and eigenvector: we assume that $\mathrm{A}$ is a real symmetric matrix of $n \times n, x$ is an n-dimensional vector, $\lambda$ is an eigenvalue of matrix $A$, and $x$ is the eigenvector corresponding to eigenvalue $\lambda$ of matrix $A$. The formula is as follows:

$$
A x=\lambda x \text {. }
$$

If we assume that the size of the $n$ eigenvalues of the matrix $\mathrm{A}$ is $\lambda_{1}>\lambda_{2}>\cdots>\lambda_{n}$ and the eigenvectors $\left(\omega_{1}, \omega_{2}, \cdots, \omega_{n}\right)$ corresponding to the $n$ eigenvalues are linearly independent, then the matrix A can be decomposed into

$$
A=W \sum W^{-1}
$$

where $\mathrm{W}$ is an $n \times n$-dimensional matrix composed of $n$ eigenvectors and $\sum$ is an $n \times n$-dimensional matrix whose $n$ eigenvalues are the main diagonal $n$ feature vectors which are standardized, namely,

$$
\begin{aligned}
\left\|\omega_{i}\right\|_{2} & =1, \\
\omega_{i}^{T} \omega_{i} & =1 .
\end{aligned}
$$

This is the orthonormal basis of $n$ eigenvectors. $W^{T} W=$ 1 is $W^{T}=W^{-1}$, so $\mathrm{W}$ is a unitary matrix.

$$
A=W \sum W^{T} \text {. }
$$

Singular value matrix factorization is applied to dimensionality reduction.

The principle of singular value matrix decomposition is to decompose the initial matrix A into the form of multiplication of the three matrices, namely, $U, \sum, V^{T}$. Among them, $\mathrm{A}$ is a matrix of $m \times n$, and $\mathrm{U}$ and $V^{T}$ are two unitary matrices of $m \times m$ and $n \times n$, respectively, namely:

$$
\begin{aligned}
& U^{T} U=1, \\
& V^{T} V=1,
\end{aligned}
$$

where $\sum$ is the matrix of $m \times n$.

$$
A=U \sum V^{T}
$$

The matrix $U, \sum, V^{T}$ is further solved: since the eigenvalue decomposition requires the decomposed matrix to be a square matrix, when solving the matrices $U$ and $V$, we must first construct a square matrix of $m \times m$ and a square matrix of $n \times n$, that is, $A A^{T}$ and $A^{T} A$.

We set $\left(A A^{T}\right) u_{i}=\lambda_{i} u_{i}$. Among them, $u_{i}=\left(u_{1}, u_{2}, u_{3}\right.$, $\left.\ldots, u_{m}\right)^{T}$. The matrix space of $\mathrm{n}$ formed by the eigenvectors corresponding to the $m \times m$ eigenvalues of $A A^{T}$ is $\mathrm{U}$.

We set $\left(A^{T} A\right) v_{i}=\lambda_{i} v_{i}$. Among them, $v_{i}=\left(v_{1}, v_{2}, v_{3}\right.$, $\left.\ldots, v_{n}\right)^{T}$. The matrix space of $\mathrm{m}$ formed by the eigenvectors corresponding to the $n \times n$ eigenvalues of $A^{T} A$ is $\mathrm{V}$.

After that, we derive the formula

$$
A=U \sum V^{T} \Rightarrow A V=U \sum \Rightarrow A v_{i}=\sigma_{i} u_{i} \Rightarrow \sigma_{i}=\frac{A v_{i}}{u_{i}} .
$$

According to this, each singular value $\sigma_{i}$ can be obtained. Further, the matrix $\sum$ can be obtained, namely,

$$
\sum=\operatorname{diag}\left(\sigma_{1}, \sigma_{2}, \cdots, \sigma_{r}\right),
$$

where $\sigma_{i}>0,(i=1,2, \cdots, r), r$ is the rank of matrix A, and $r=\operatorname{rank}(A)$, as shown in Figure 1 .

Generally, the sum of the first $k$ singular values accounts for more than $90 \%$ of the sum of all singular values, so the eigenvectors corresponding to the first $k$ singular values can be used to describe the original matrix. It not only reduces the dimensionality of the original matrix but also retains most of the information of the original matrix. The formulas for matrix decomposition and dimensionality reduction are as follows:

$$
A_{m \times n}=U_{m \times n} \sum_{m \times n} V_{m \times n}^{T} \approx U_{m \times k} \sum_{k \times k} V_{k \times n}^{T}
$$

where $k$ is a number smaller than $n$, which is the principle of singular value matrix dimensionality reduction. Figure 2 shows the image of singular value dimensionality reduction.

\section{Recommendation Algorithm Based on Matrix Factorization}

In the recommendation system, when SVD is used to predict ratings, the original data matrix is decomposed into the matrices $p_{u}$ and $q_{i}$ by training the user rating information. $p_{u(i)}=\left(i_{1}, i_{2}, \ldots, i_{k}\right)^{T}$ is a column of $p_{u}$, which represents the user's potential interest measure. $q_{i(j)}=\left(j_{1}, i_{2}, \ldots, j_{k}\right)^{T}$ is a column of $q_{i}$, which represents the attribute measurement of the item. $\mathrm{k}$ is the number of hidden factors set during the matrix decomposition process. The SVD scoring model is as follows:

$$
\widehat{r}_{u, i}=q_{i}^{T} p_{u}
$$

where $\widehat{r}_{u, i}$ is the prediction score item. The Netflix contest that started in 2006 aroused people's interest in recommendation systems. Among them, the team headed by Koren conducted in-depth research on SVD matrix factorization and further proposed a new matrix factorization algorithm Biased-SVD. The algorithm adds consideration of system characteristics, project characteristics, and user characteristics. The BiasedSVD scoring model is as follows [19]:

$$
\widehat{r}_{u, i}=\mu+b_{u}+b_{i}+q_{i}^{T} p_{u},
$$

where $\mu$ is the average system scoring, $b_{u}$ is the user scoring bias, and $b_{i}$ is the item scoring bias. The $S V D++$ algorithm is an improvement of the Biased-SVD matrix decomposition. It incorporates information about other items evaluated by users on the basis of the Biased-SVD algorithm. The $S V D++$ scoring model is as follows: 


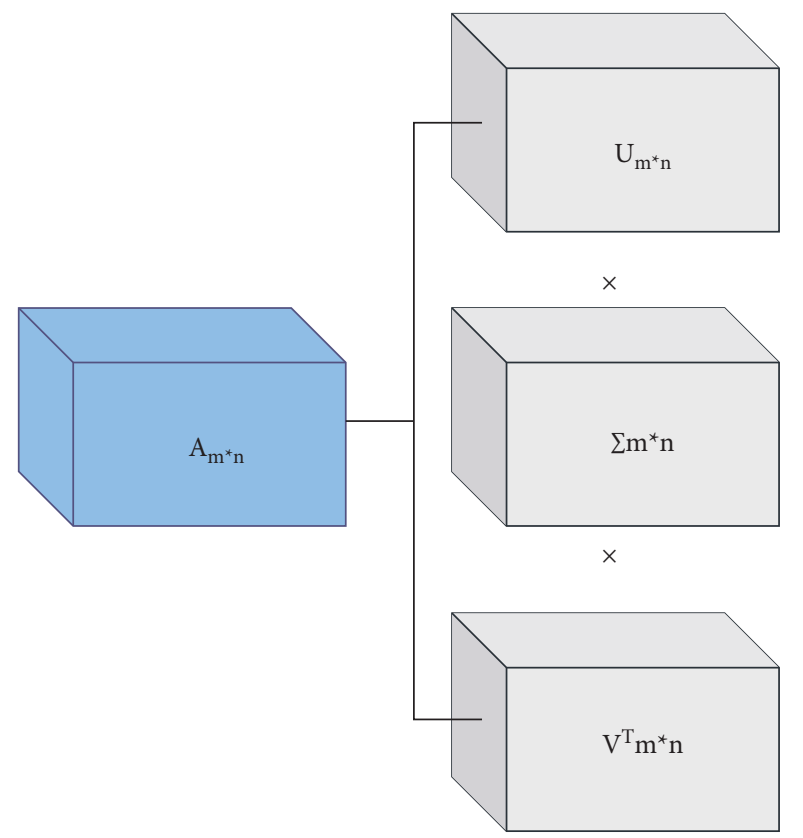

Figure 1: Principle of matrix decomposition.

$$
\widehat{r}_{u, i}=\mu+b_{u}+b_{i}+q_{i}^{T}\left(p_{u}+|R(u)|^{\frac{1}{2}} \sum_{j \in R(u)} y_{j}\right),
$$

where $R(u)$ is the set of items evaluated by user $u$ and $y_{j}$ is the attribute vector of the item. $d_{-} S V D++$ is a dual model of $S V D++$, that is, matrix decomposition based on implicit user feedback. The $d_{-} S V D++$ scoring model is as follows [19]:

$$
\widehat{r}_{u, i}=\mu+b_{u}+b_{i}+p_{u}^{T}\left(q_{i}+|N(i)|^{-\frac{1}{2}} \sum_{v \in N(i)} x_{v}\right)
$$

where $N(i)$ is the set of users who have evaluated item $i$ and $x_{v}$ is the attribute vector of users.

The principle of predictive scoring is as follows.

First, according to the given prediction score model $S V D++$, the error between the predicted score and the true score is calculated:

$$
e_{u, i}=r_{u, i}-\widehat{r}_{u, i}
$$

The loss function is a statistical method of error, which uses the sum of squares to calculate the error. In the recommendation system, the size of the loss function reflects the gap between the predicted score and the true score. In general, the difference between the projected and actual score should be as little as feasible. However, if the loss function's value is too small, it will result in overfitting between the trained model and the training set data, lowering the model's generalisation performance and lowering its practical usefulness. In order to assure the model's generalisation capabilities, a regular term is included in the loss function computation. The following is the loss function [20]:

$$
S S E=\frac{1}{2} \sum_{u, i} e_{u, i}^{2}+\frac{1}{2} \lambda\left\|p_{u}\right\|^{2}+\frac{1}{2} \lambda\left\|q_{i}\right\|^{2}+\frac{1}{2} \lambda\left\|b_{u}\right\|^{2}+\frac{1}{2} \lambda\left\|b_{i}\right\|^{2}+\frac{1}{2} \sum_{j \in R(u)}\left\|y_{j}\right\|^{2},
$$

where $\lambda$ is the regularization factor and $\left\|p_{u}\right\|^{2},\left\|q_{i}\right\|^{2},\left\|b_{u}\right\|^{2},\left\|b_{i}\right\|^{2},\left\|y_{j}\right\|^{2}$ represent the regularization term.

This article uses stochastic gradient descent (SGD), and Figure 3 shows the SGD process. When SGD updates parameters, it updates each parameter every time a sample is added. Due to the faster update speed, this can reduce the time for training parameters. Since SGD only uses a sample of data during the update process, it will cause certain fluctuations during the update. However, due to the existence of such fluctuations, it is easier to jump out of the local optimum and find the global optimum. The following formula is the principle of gradient update [21]:

$$
\theta_{i}^{n+1}=\theta_{i}^{n}-\alpha \frac{\partial S S E}{\partial \theta_{i}}
$$

where $\alpha$ is the learning rate and $\theta_{i}$ is the model parameter.

The parameters are differentiated:

$$
\left\{\begin{array}{l}
\frac{\partial S S E}{\partial p_{u}}=-e_{u, i} q_{i}+\lambda p_{u} \\
\frac{\partial S S E}{\partial q_{i}}=-e_{u, i}\left(p_{u}+|R(u)|^{-1 / 2} \sum_{j \in R(u)} y_{j}\right)+\lambda q_{i} \\
\frac{\partial S S E}{\partial b_{u}}=-e_{u, i}+\lambda b_{u} \\
\frac{\partial S S E}{\partial b_{i}}=-e_{u, i}+\lambda b_{i} \\
\frac{\partial S S E}{\partial y_{j}}=-e_{u, i}|R(u)|^{-1 / 2} q_{i}+\lambda y_{j} j \in R(u)
\end{array}\right.
$$

The parameters are updated: 


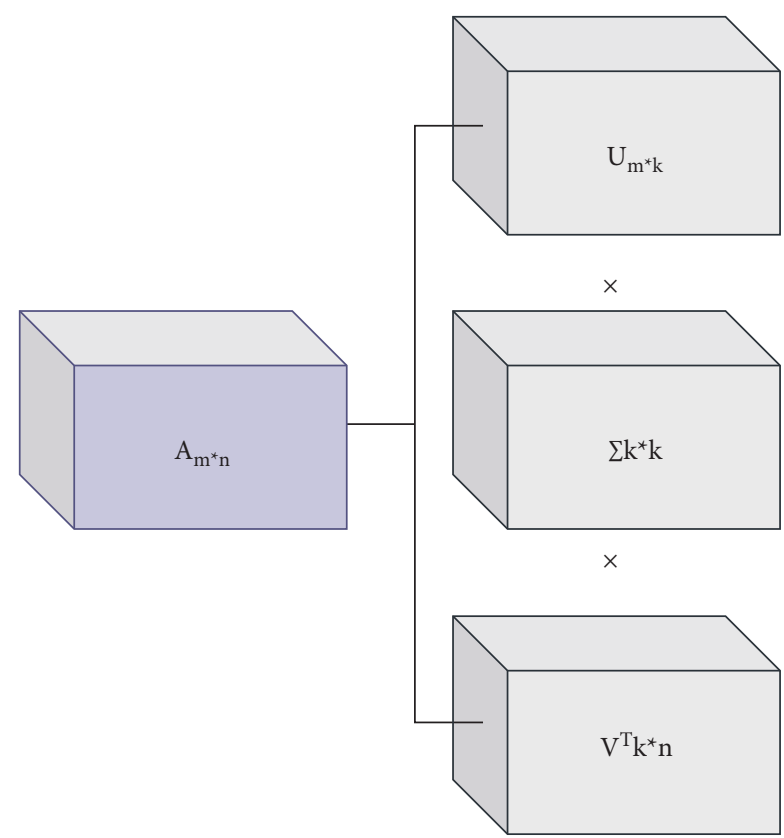

FIGURE 2: The principle of matrix factorization for dimensionality reduction.

$$
\left\{\begin{array}{l}
p_{u+1}=p_{u}-\eta \frac{\partial S S E}{\partial p_{u}} \\
q_{i+1}=q_{i}-\eta \frac{\partial S S E}{\partial q_{i}} \\
b_{u+1}=b_{u}-\eta \frac{\partial S S E}{\partial b_{u}} \\
b_{i+1}=b_{i}-\eta \frac{\partial S S E}{\partial b_{i}} \\
y_{j+1}=y_{j}-\eta \frac{\partial S S E}{\partial y_{j}} j \in R(u)
\end{array}\right.
$$

where $\eta$ is the learning rate, which is used to control the speed of gradient descent. If the gradient descent speed is too fast, it may cause oscillations, which is not conducive to the convergence of the algorithm. However, if the speed of the gradient descent is too slow, it will double the training time of the algorithm and reduce the use value of the algorithm.

The trained parameters are used in the $S V D++$ prediction scoring model. When a user and an item to be scored are entered, the system will perform a predictive score. The prediction scoring process of the $d_{-} S V D++$ algorithm is roughly the same as that of the $S V D++$ algorithm, but the emphasis is different. The $S V D++$ algorithm focuses on the impact of item implicit feedback on the predicted score, while the $d_{-} S V D++$ algorithm focuses on the impact of user implicit feedback on the predicted score [22].

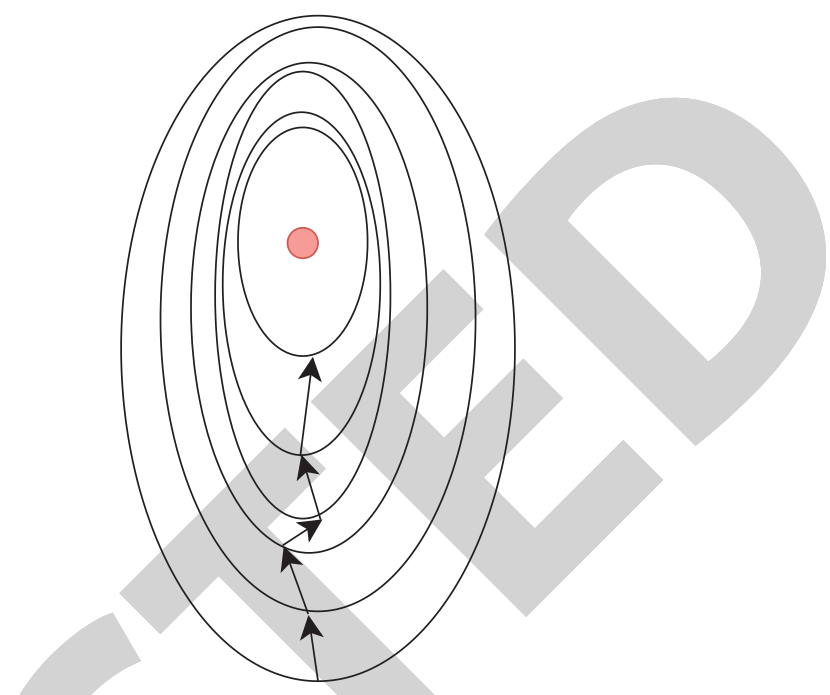

FIGURE 3: The process of stochastic gradient descent.

\section{Construction of Recommendation System}

The semantic neighborhood and the neighborhood recommended by the traditional collaborative filtering algorithm are merged based on the experimental ratio. The similarity calculated by the constructed semantic similarity matrix and the knowledge similarity matrix are then combined to form the recommended list. The following is the flow of the algorithm. To create an entity comparison table, the knowledge in the evaluation dataset is first mapped to the entities in the built knowledge graph. The semantic similarity following the entity disambiguation procedure is then utilised to produce a semantic neighborhood set by constructing a semantic similarity matrix. Following that, the evaluation matrix is utilised to determine the similarity between the knowledge, and the knowledge's similarity matrix is produced, resulting in the knowledge's neighborhood set. Finally, the algorithm chooses an acceptable fusion ratio, merges the semantic and knowledge neighborhood sets to some extent, and generates the knowledge recommendation list. Figure 4 depicts the stages of the algorithm [23].

Domain ontology gives a formal description of the concepts and relationships of entities in the domain and the characteristics and laws of the domain. When constructing domain ontology, we must first grasp the needs of the domain and clarify the purpose, use, and users of domain ontology construction. This article takes the entrepreneurial field as an example, proposes a method of constructing ontology in this field, and completes the construction of ontology in this field. The steps are as follows. The first step is to determine the field of scientific and technological papers in the field of entrepreneurship as the field of the ontology of this paper. Then, we obtain the required data in this field. The second step is to construct the ontology. The main work is to define the core concepts of the ontology, define the concept hierarchy and conceptual attributes, and finally realize the formal coding of the ontology. The third step is to instantiate the ontology and then fill the pattern layer with 


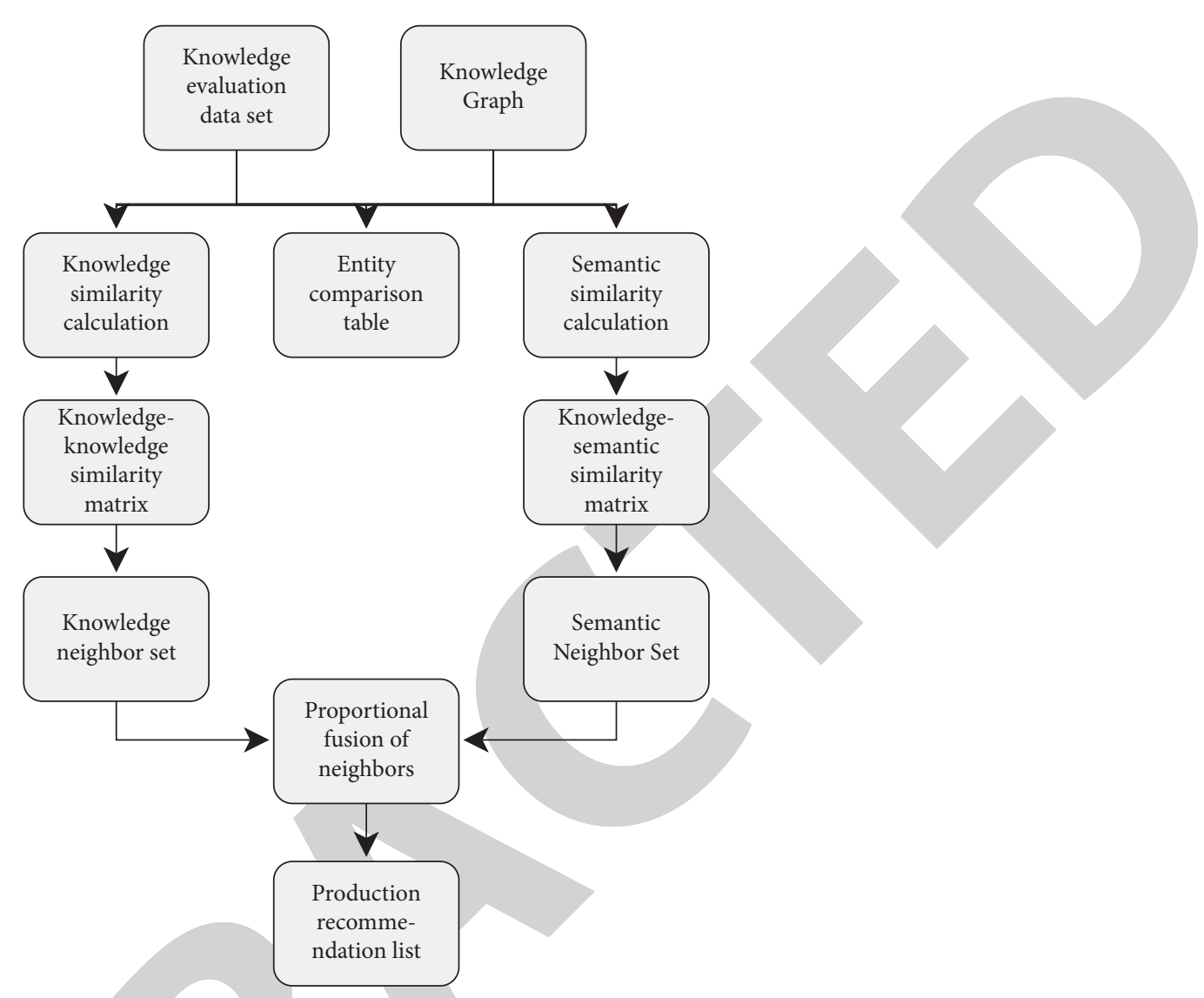

FIGURE 4: Diagram of algorithm steps.

data, which is the data layer of the knowledge graph. Figure 5 shows the process of constructing the ontology of scientific papers in the field of entrepreneurship [24].

This paper proposes the KG-GCN-LSR model, which is an end-to-end trainable framework. The KG-GCN-LSR model uses a knowledge graph to capture the relationship between items to achieve better recommendations. The KGGCN-LSR model first applies a trainable function to calculate the project embedding of a specific user and uses the graph convolutional neural network to spread and aggregate the surrounding domain node information of the corresponding entity of the project on the knowledge graph. However, the number of neighbor entities around an entity is often very large, and it is also very difficult to aggregate entity features. Therefore, we use the idea of graph convolutional neural network (GCN) to aggregate features of neighbor entities with a fixed size [25].

The core idea of GCN is to learn to use neural networks to iteratively aggregate feature information from the local map domain. The feature of each node's neighbor node is propagated to the node for weighted summation to get the aggregate feature of the point. Then, we directly use this aggregated feature value as the feature value to be propagated to the next layer. We first sample a fixed-size neighbor set as the receptive field of each node, that is, the area where the network features can see the input graph. In the KGGCN-LSR network model, we also adopt a fixed-size neighbor set as the receptive field of each entity. This will control the calculation cost of KG-GCN-LSR to a certain range and can improve the scalability of the recommendation algorithm to a certain extent. The KG-GCN model diagram is shown in Figure 6.

\section{System Performance Verification}

This study examines the model's performance once it has been constructed. This paper's recommendation model is mostly employed in the education business, and it can offer individualised information that is appropriate for students' learning. As a result, the model is primarily validated in terms of system performance and student satisfaction in this research. First and foremost, this study scores system performance and evaluates performance using expert scoring methods. There are a total of 66 datasets that will be evaluated. Table 1 and Figure 7 illustrate the outcomes.

It is not difficult to see from the above chart that the model constructed in this paper performs well. Next, this paper analyzes the practical effect of the model through the actual experience of the students. The results are shown in Table 2 and Figure 8.

It is not difficult to see from Table 2 and Figure 8 that the practical effect of the model constructed in this paper is very significant, so it can be applied to actual teaching. 


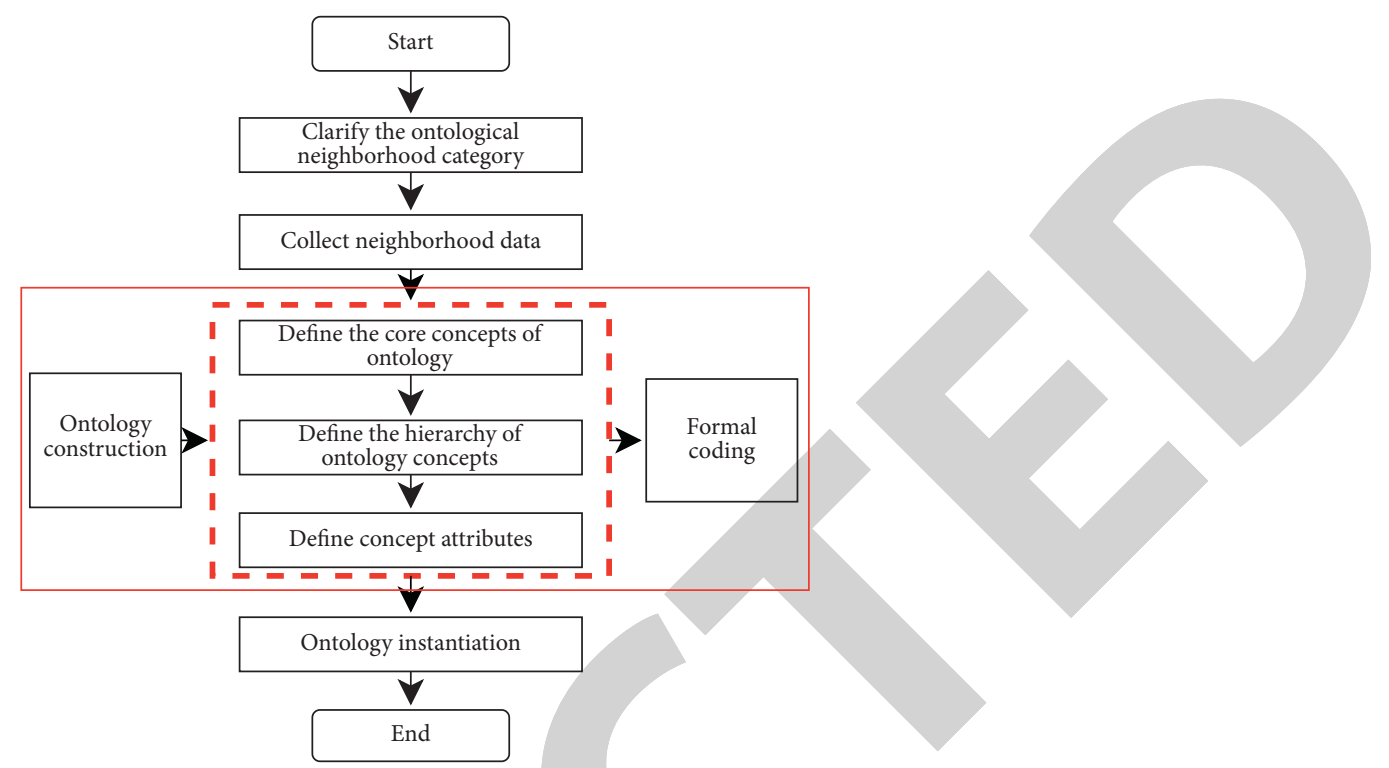

Figure 5: The flowchart of knowledge ontology construction.

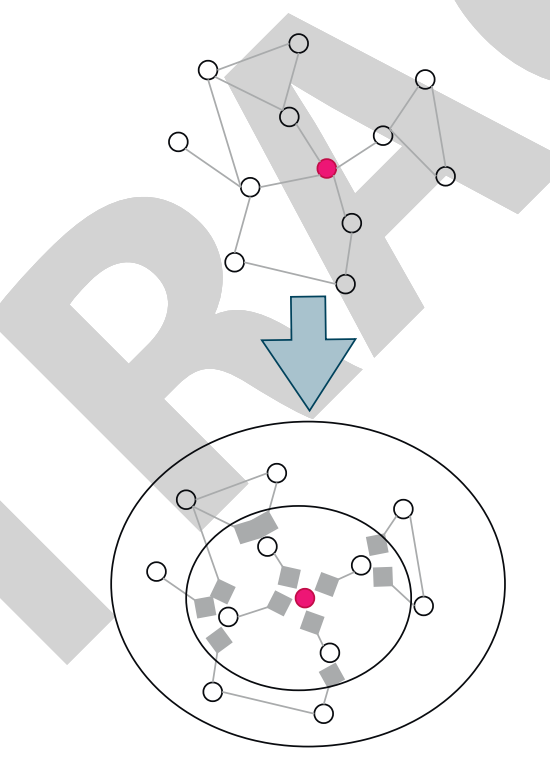

FigURE 6: KG-GCN model diagram.

TABLE 1: Evaluation results of system performance.

\begin{tabular}{|c|c|c|c|c|c|}
\hline No. & Scores & No. & Scores & No. & Scores \\
\hline 1 & 95.3 & 29 & 93.8 & 57 & 94.1 \\
\hline 2 & 95.9 & 30 & 93.2 & 58 & 96.8 \\
\hline 3 & 93.7 & 31 & 94.8 & 59 & 94.9 \\
\hline 4 & 93.1 & 32 & 95.0 & 60 & 93.2 \\
\hline 5 & 94.5 & 33 & 96.0 & 61 & 93.8 \\
\hline 6 & 95.7 & 34 & 95.4 & 62 & 95.1 \\
\hline 7 & 96.0 & 35 & 95.5 & 63 & 94.2 \\
\hline 8 & 93.3 & 36 & 94.0 & 64 & 94.7 \\
\hline 9 & 95.0 & 37 & 95.4 & 65 & 94.1 \\
\hline 10 & 95.1 & 38 & 95.3 & 66 & 96.2 \\
\hline 11 & 95.1 & 39 & 93.3 & 67 & 95.2 \\
\hline 12 & 96.8 & 40 & 96.5 & 68 & 95.8 \\
\hline 13 & 94.6 & 41 & 96.8 & 69 & 95.6 \\
\hline 14 & 93.5 & 42 & 93.0 & 70 & 96.7 \\
\hline 15 & 95.6 & 43 & 94.9 & 71 & 93.1 \\
\hline
\end{tabular}


TABle 1: Continued.

\begin{tabular}{|c|c|c|c|c|c|}
\hline No. & Scores & No. & Scores & No. & Scores \\
\hline 16 & 95.5 & 44 & 96.6 & 72 & 96.9 \\
\hline 17 & 93.7 & 45 & 96.4 & 73 & 93.7 \\
\hline 18 & 93.5 & 46 & 96.4 & 74 & 94.1 \\
\hline 19 & 94.6 & 47 & 95.2 & 75 & 93.7 \\
\hline 20 & 93.6 & 48 & 94.9 & 76 & 95.0 \\
\hline 21 & 93.1 & 49 & 94.2 & 77 & 93.6 \\
\hline 22 & 96.2 & 50 & 94.3 & 78 & 96.9 \\
\hline 23 & 94.1 & 51 & 96.4 & 79 & 95.0 \\
\hline 24 & 93.3 & 52 & 96.3 & 80 & 95.7 \\
\hline 25 & 95.3 & 53 & 95.0 & 81 & 93.3 \\
\hline 26 & 96.6 & 54 & 94.9 & 82 & 96.3 \\
\hline 27 & 96.3 & 55 & 93.5 & 83 & 96.9 \\
\hline 28 & 94.7 & 56 & 96.5 & 84 & 95.2 \\
\hline
\end{tabular}

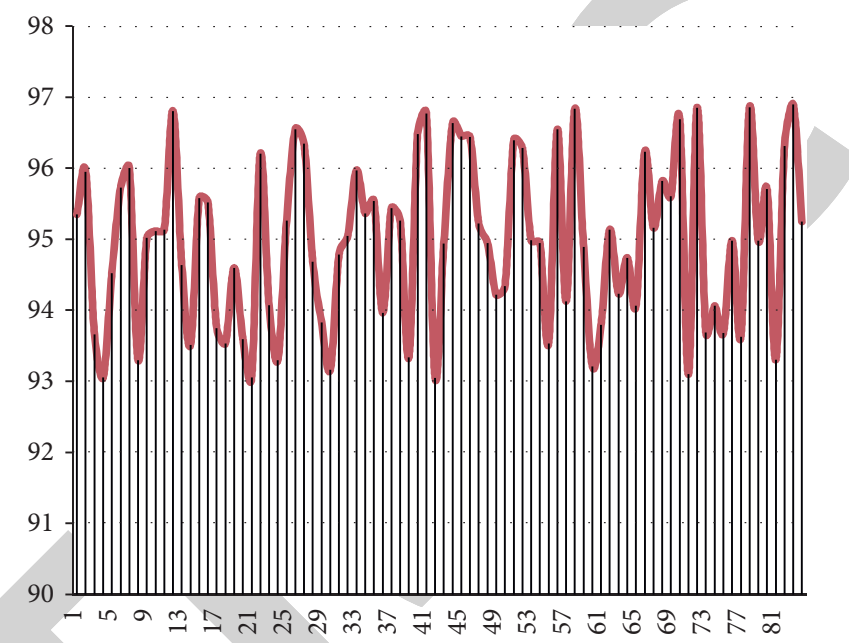

FIgURE 7: Statistical diagram of evaluation results of system performance.

TABLE 2: Scoring of model practice effect.

\begin{tabular}{|c|c|c|c|c|c|}
\hline $\mathrm{NO}$ & Scores & $\mathrm{NO}$ & Scores & $\mathrm{NO}$ & Scores \\
\hline 1 & 86 & 29 & 80 & 57 & 85 \\
\hline 2 & 89 & 30 & 95 & 58 & 92 \\
\hline 3 & 77 & 31 & 82 & 59 & 92 \\
\hline 4 & 92 & 32 & 89 & 60 & 90 \\
\hline 5 & 94 & 33 & 86 & 61 & 83 \\
\hline 6 & 84 & 34 & 90 & 62 & 86 \\
\hline 7 & 73 & 35 & 81 & 63 & 73 \\
\hline 8 & 78 & 36 & 78 & 64 & 81 \\
\hline 9 & 85 & 37 & 82 & 65 & 88 \\
\hline 10 & 90 & 38 & 92 & 66 & 88 \\
\hline 11 & 87 & 39 & 76 & 67 & 86 \\
\hline 12 & 84 & 40 & 86 & 68 & 82 \\
\hline 13 & 82 & 41 & 88 & 69 & 94 \\
\hline 14 & 84 & 42 & 93 & 70 & 90 \\
\hline 15 & 78 & 43 & 77 & 71 & 94 \\
\hline 16 & 85 & 44 & 83 & 72 & 80 \\
\hline 17 & 88 & 45 & 81 & 73 & 94 \\
\hline 18 & 93 & 46 & 93 & 74 & 94 \\
\hline 19 & 91 & 47 & 83 & 75 & 73 \\
\hline 20 & 75 & 48 & 78 & 76 & 77 \\
\hline 21 & 77 & 49 & 76 & 77 & 81 \\
\hline 22 & 83 & 50 & 87 & 78 & 95 \\
\hline
\end{tabular}


TABLE 2: Continued.

\begin{tabular}{lccccc}
\hline NO & Scores & NO & Scores & NO & Scores \\
\hline 23 & 88 & 51 & 85 & 79 & 80 \\
24 & 88 & 52 & 90 & 81 & 72 \\
25 & 76 & 53 & 91 & 82 & 81 \\
26 & 84 & 54 & 86 & 83 & 82 \\
27 & 74 & 55 & 79 & 84 & 74 \\
28 & 76 & 56 & 83 & 93 \\
\hline
\end{tabular}

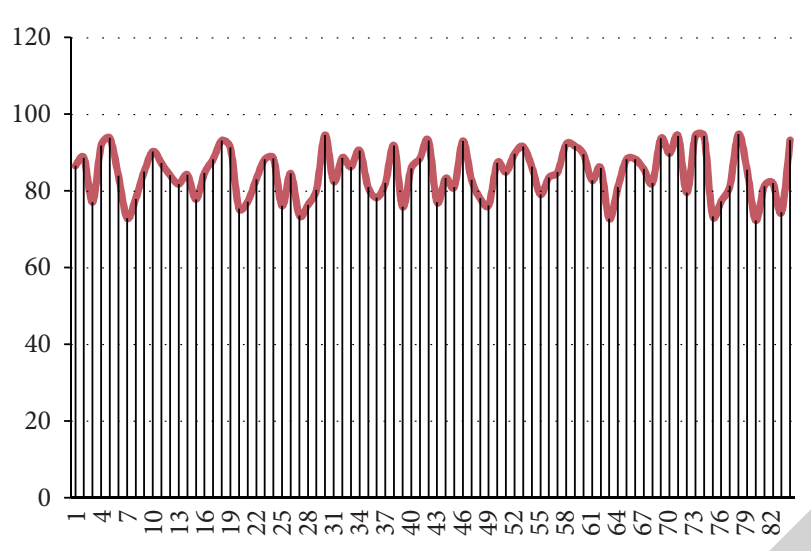

FIgURE 8: Statistical diagram of scoring of model practice effect.

\section{Conclusion}

In the era of big data, while enjoying the benefits of data, people also experience the troubles caused by information overload. In order to solve the problems caused by information overload, people put forward the concept of recommendation systems. Nowadays, recommendation systems are involved in all major industries. They not only improve people's efficiency in obtaining useful information but also promote the development of enterprises. This paper conducts a specific research on the application of matrix factorization in the education recommendation system with IoT networks. Moreover, this paper not only discovers the shortcomings of matrix factorization used to predict scoring but also proposes corresponding improvement measures. Finally, experimental verification shows that the algorithm proposed in this paper effectively improves the accuracy of the prediction score. This article first outlines, classifies, and summarizes the recommendation system and then introduces the specific research object of this paper, that is, the matrix decomposition recommendation algorithm. At present, most of the recommendation algorithms based on matrix factorization have the problem that the implicit feedback information of users and items is considered single and the feedback information is directly used without measuring the relevance of the feedback information. Moreover, they have problems such as inaccurate calculation of the correlation between user and project information and sparse data .

\section{Data Availability}

The data used to support the findings of this study are included within the article.

\section{Conflicts of Interest}

The authors declare that they have no conflicts of interest.

\section{Acknowledgments}

This study was supported by the Educational Department of Jilin Province (the research of English online class of high vocational college on political character of curriculum) (2020ZCY349).

\section{References}

[1] I. Viktoratos, A. Tsadiras, and N. Bassiliades, "Combining community-based knowledge with association rule mining to alleviate the cold start problem in context-aware recommender systems," Expert Systems with Applications, vol. 101, no. JUL, pp. 78-90, 2018.

[2] M. Li, M. Yuan, and Y. Xu, "An approach to task-oriented knowledge recommendation based on multi-granularity fuzzy linguistic method," Kybernetes, vol. 44, no. 3, pp. 460-474, 2015.

[3] T. Qiu, T. Y. Han, L. X. Zhong, Z. K. Zhang, and G. Chen, "Redundant correlation effect on personalized recommendation," Computer Physics Communications, vol. 185, no. 2, pp. 489-494, 2014.

[4] W. Zhou and W. Han, "Personalized recommendation via user preference matching," Information Processing \& Management, vol. 56, no. 3, pp. 955-968, 2019.

[5] J. Mao, K. Lu, G. Li, and M. Yi, "Profiling users with tag networks in diffusion-based personalized recommendation," Journal of Information Science, vol. 42, no. 5, pp. 711-722, 2016.

[6] Y. Zuo, M. Gong, J. Zeng, L. Ma, and L. Jiao, "Personalized recommendation based on evolutionary multi-objective optimization," IEEE Computational Intelligence Magazine, vol. 10, no. 1, pp. 52-62, 2015.

[7] G. Costa and R. Ortale, "Model-based collaborative personalized recommendation on signed social rating networks," ACM Transactions on Internet Technology, vol. 16, no. 3, pp. 1-21, 2016.

[8] Y. Guan, Q. Wei, and G. Chen, "Deep learning based personalized recommendation with multi-view information integration," Decision Support Systems, vol. 118, no. MAR., pp. 58-69, 2019.

[9] C. Yang, Q. Fan, and T. Wang, "RepoLike: a multi-featurebased personalized recommendation approach for opensource repositories," Frontiers of Information Technology \& Electronic Engineering, vol. 20, no. 02, pp. 86-101, 2019.

[10] Q. Lin, X. Wang, B. Hu et al., "Multiobjective personalized recommendation algorithm using extreme point guided evolutionary computation," Complexity, vol. 2018, no. 3, 18 pages, Article ID 1716352, 2018. 
[11] X. U. Dazhi, "Research on music culture personalized recommendation based on factor decomposition machine," Personal and Ubiquitous Computing, vol. 24, no. 2, pp. 247257, 2020.

[12] N. X. Bach, N. D. Hai, and T. M. Phuong, "Personalized recommendation of stories for commenting in forum-based social media," Information Sciences, vol. 352-353, no. 2, pp. 48-60, 2016.

[13] D. Guo, Y. Zhu, W. Xu, S. Shang, and Z. Ding, "How to find appropriate automobile exhibition halls: towards a personalized recommendation service for auto show," Neurocomputing, vol. 213, no. NOV.12, pp. 95-101, 2016.

[14] C. Guilin, Z. Xuzhen, and Y. Zhao, "Personalized recommendation via suppressing excessive diffusion," Mathematical Problems in Engineering, vol. 2017, no. 1, 10 pages, Article ID 2587069, 2017.

[15] O. Kassak, M. Kompan, and M. Bielikova, "User preference modeling by global and individual weights for personalized recommendation," Acta Polytechnica Hungarica, vol. 12, no. 8, pp. 27-41, 2015.

[16] U. Liji, Y. Chai, and J. Chen, "Improved personalized recommendation based on user attributes clustering and score matrix filling," Computer Standards \& Interfaces, vol. 57, no. 5, pp. 59-67, 2018.

[17] T. Ha and S. Lee, "Item-network-based collaborative filtering: a personalized recommendation method based on a user's item network," Information Processing \& Management, vol. 53, no. 5, pp. 1171-1184, 2017.

[18] W. Intayoad, T. Becker, and P. Temdee, "Social context-aware recommendation for personalized online learning," Wireless Personal Communications, vol. 97, no. 1, pp. 1-17, 2017.

[19] J. Xia, G. Li, and Z. Cao, "Personalized exercise recommendation algorithm combining learning objective and assignment feedback," Journal of Intelligent and Fuzzy Systems, vol. 35, no. 3, pp. 2965-2973, 2018.

[20] V. Subramaniyaswamy, G. Manogaran, and R. Logesh, "An ontology-driven personalized food recommendation in IoTbased healthcare system," The Journal of Supercomputing, vol. 75 , no. 2, pp. 1-33, 2018.

[21] K. Kourou, T. P. Exarchos, K. P. Exarchos, M. V. Karamouzis, and D. I. Fotiadis, "Machine learning applications in cancer prognosis and prediction," Computational and Structural Biotechnology Journal, vol. 13, no. 5, pp. 8-17, 2015.

[22] S. Amershi, M. Cakmak, W. B. Knox, and T. Kulesza, "Power to the people: the role of humans in interactive machine learning," AI Magazine, vol. 35, no. 4, pp. 105-120, 2014.

[23] V. Rodriguez-Galiano, M. Sanchez-Castillo, M. Chica-Olmo, and M. Chica-Rivas, "Machine learning predictive models for mineral prospectivity: an evaluation of neural networks, random forest, regression trees and support vector machines," Ore Geology Reviews, vol. 71, no. 3, pp. 804-818, 2015.

[24] C. W. Coley, R. Barzilay, T. S. Jaakkola, W. H. Green, and K. F. Jensen, "Prediction of organic reaction outcomes using machine learning," ACS Central Science, vol. 3, no. 5, pp. 434-443, 2017.

[25] A. Chowdhury, E. Kautz, B. Yener, and D. Lewis, "Image driven machine learning methods for microstructure recognition," Computational Materials Science, vol. 123, no. 8, pp. 176-187, 2016. 